\title{
Multiparametric Quantitative MRI as a Metric for Radiation Treatment Planning
}

\author{
Ali Fatemi* PhD, Claus Chunli Yang PhD, Bart Morris MS, Madhava R Kanakamedala MD, Somayeh Taghizadeh MS, \\ Srinivasan Vijayakumar MD \\ Department of Radiology and Radiation Oncology, University of Mississippi Medical Center, USA
}

*Corresponding author: Ali Fatemi, Assistant professor Departments of Radiology and Radiation Oncology, University of Mississippi Medical Center, USA

Submission: :

\begin{abstract}
Magnetic resonance imaging (MRI) provides excellent soft tissue contrast, and in combination with its quantitative functional imaging capability, this modality is ideal for use in radiotherapy. MRI images, either used directly or fused with CT, play an increasingly important role in contouring gross tumor volume (GTV) and organs at risk (OAR) in radiation treatment planning (RTP) systems. The soft tissue contrast of MRI images provides more accurate tumor delineation than CT, although CT images have sufficient geometrical stability and electron density information for accurate radiation treatment planning. Many vendors now offer $70 \mathrm{~cm}$ wide-bore MRI systems with dedicated radiofrequency (RF) coils and immobilization devices for RTP simulation comparable to CT simulators.
\end{abstract}

Many groups are researching future applications of MRI simulation independent of CT and as a stand-alone modality for RTP and online guided radiotherapy. The aim of this paper is to discuss the major functional MRI pulse sequences used in RTP: dynamic contrast enhanced MRI (DCE-MRI), diffusion-weighted imaging (DWI) and magnetic resonance spectroscopic imaging (MRSI). We discuss the potential of these functional MRI techniques in different sites for tumor response evaluation and possible adaptive radiotherapy.

Keywords: MRI, Adaptive radiation therapy, MRI guided radiation therapy, quantitative imaging

Abbreviations: MRI: Magnetic Resonance Imaging; ADC: Apparent Diffusion Coefficient; GTV: Gross Tumor Volume; OAR: Organs at Risk; RTP: Radiation Treatment Planning; UTE: Using ultra-Short Echo; TPS: Treatment Planning System; RF: Radiofrequency; DCE-MRI: Dynamic Contrast enhanced MRI; DWI: Diffusion Weighted Imaging; MRSI: Magnetic Resonance Spectroscopic Imaging; DRRs: Digitally Reconstructed Radiographs; HDR: High Dose radiation rate IMRT: Intensity Modulated Radiation Therapy; VMAT: Volumetric Modulated Arc Therapy; FSRT: Fractionated Stereotactic Radiotherapy; SRS: Stereotactic Radiosurgery; RT: Radiotherapy; TCP: Tumor Control Probability; CT: Computed Tomography; IGRT: Image Guided Radiation Therapy; TWIST: Time Resolved Angiography with Interleaved Stochastic Trajectories; MRA: Magnetic Resonance Angiography; ROI: Regions of Interest; ADC: Apparent Diffusion Coefficient; 1H: Hydrogen; 31P: Phosphorus -31; 19F: Fluorine-19; 13C: Carbon-13; SVS: Single Voxel Spectroscopy; CSI: Chemical Shift Imaging; NAA: N-Acetylaspartate; FRT: Fractionated Radiotherapy; GBM: Glioblastoma; FLAIR: Fluidattenuated Inversion Recovery; AVMs: Arteriovenous Malformations; PDV: Prescribed 95\% Isodose Volume; PRM: Parametric Response Map; PFS: Progression Free Survival; PCA: Principle Component Analysis; BOLD: Blood Oxygen Level Dependent; USPIO: Ultra Small Particle Iron Oxides; CBV: Cerebral Blood Volume; CBF: Cerebral Blood Flow; MIT: Mean Transit Time; SNR: Signal to Noise Ratio

\section{Introduction}

Conformal radiation therapy, intensity-modulated radiation therapy (IMRT), volumetric modulated arc therapy (VMAT), and stereotactic radiosurgery are offering improved sparing of normal tissue and escalation of tumor dose for better tumor control probability (TCP). We will discuss MRI capability as a superior soft tissue contrast modality for Radiation treatment planning (RTP) and guided radiotherapy.

Using MRI directly in RTP allows realization of the full potential of advanced radiation therapy delivery systems and techniques using the superior anatomical and functional imaging of MRI at the contouring stage, and ideally, on MRI-linac machines, to confirm patient setup and provide real-time accurate tumor targeting.

In current practice, we are using computed tomography (CT) for radiation treatment planning simulations. CT uses ionizing radiation, with the concomitant risk of indication of a secondary malignancy [1]. These risks could potentially be increased, especially for adaptive replanning. MRI does not use ionizing radiation and the only biohazard is exposure to radiofrequency, which is always controlled and is well defined with the current advances in RF power and coil designs. MRI uses a static magnetic field (B0) and dynamic gradient magnetic fields $\left(G_{z^{\prime}}, G_{x^{\prime}}\right.$ and $\left.G_{y}\right)$ to localize the magnetic signal with high anatomical spatial resolution and superior contrast. In addition, using functional MRI sequences allows extraction of information about tumor perfusion vascular permeability, metabolic status, cellularity, magnetic susceptibility (texture) and hypoxia [2].

In recent years several new MRI-guided radiation therapy systems have been introduced, including MRIdian (ViewRay ${ }^{\mathrm{TM}}$ ) and MRI-linac prototypes. MRI is a main component of new 
systems being used in online image guided radiotherapy (IGRT) $[3,4]$. The engineering of such systems is challenging, especially with the possibility of RF and main field interactions between linac and MRI machines respectively. Current designs minimize possible RF perturbation from linac and keep the magnetic field local while maintaining the sensitive parts of the linac in zero field. During treatment planning the electron return effect needs to be considered at the air-lung-tissue interface $[5,6]$.

MRI signal is strongly dependent on the abundance of hydrogen atoms in the human body (primarily as water and fat). MR image contrast is predominantly dependent on the longitudinal (T1) or transverse (T2) relaxation times of fat and water. The variation of T1 (or T2) among tissues is the basis for tissue contrast in MRI images. In general, T1-weighted images possess a higher signal to noise ratio (SNR) but require a longer scanning time. This image provides better geometrical and anatomical tumor and normal tissue boundary delineation. T2-weighted images are more sensitive to biological characteristics and provide more information to differentiate tumor from healthy tissue. There are many applications of T2-weighted images for diagnosis and localization of prostate, head and neck and breast tumors $[7,8]$. One of the main challenges of using MRI in radiation oncology is that MRI signal is not directly related to tumor tissue properties. The same limitation is also applicable to CT images, where image contrast is directly dependent on the tissue x-ray attenuation coefficient $[9,10]$. On the other hand, MRI can provide more functional information, such as tumor post resound than is possible with CT; we call these techniques "quantitative function MRI" [11-13]. This review article will focus on current applications of MRI imaging in RTP. We discuss different MRI pulse sequences and their potential in various cancer-treatment sites. We hope that this discussion will open up new opportunities for clinical trials and introduce new tools for better cancer management. Below, we outline some current and advanced multiparametric quantitative MRI techniques used in radiation treatment planning and general radiotherapy.

\section{Dynamic Contrast-Enhanced MRI (DCE-MRI)}

DCE-MRI has been proven clinically useful to diagnose various tumors, and to serve an important role in post-treatment (radiotherapy or chemotherapy) tumor response evaluation [2]. DCE-MRI signal is dependent on the type of contrast agent employed (usually a gadolinium-based chelate). The gadolinium (Gd) agent shortens the relaxation time (T1); therefore, the region with high Gd concentration shows hypointense signal and can be differentiated from surrounding tissue. Briefly, during the first run the regular pre-contrast T1-weighted scan is collected without $\mathrm{Gd}$, then $\mathrm{Gd}$ is injected $\left(0.05-1 \mathrm{mmol} \cdot \mathrm{kg}^{-1}\right)$. After sometime a postcontrast T1-weighed scan is collected. Most vendors provide postprocessing tools to analyze DCE-MRI data. Basically, by calculating T1 differences between each voxel pre-and post-contrast, it is possible to map out permeable blood vessels and corresponding tumor tissue.

This approach is predicated on the principle that the Gd agent re-enters blood vessels more rapidly in areas of healthy cells and high cell density since it cannot pass through the cell membranes. In damaged tissues or tissues with a lower cell density, the gadolinium lingers in the extracellular space.

Temporal resolution is a key feature for DCE-MRI pulse sequences. It is recommended to use a fast gradient echo T1weighted 2D sequence with temporal resolution of 2-10 s, or $30 \mathrm{~s}$ for 3D volumetric imaging.

\section{Dynamic susceptibility contrast (DSC) MR perfusion}

This technique relies on prolonging $\mathrm{T}^{*}$ and signal avoid on T2*-weighed images with or without contrast agents. The most common parameters calculated with this method are cerebral blood volume (rCBV), cerebral blood flow ( $\mathrm{rCBF}$ ), and mean transit time (MIT). This technique can be run in cine mode to study multiple arterial, mixed, or venous phases for magnetic resonance angiography (MRA).

After intravenous injection of a Gd-based contrast agent, a series of rapid scans is performed at the same region of interest (ROI) during the first pass. This generates a batch of images, with the signal in each voxel representing the effect of contrast agent on T2/T2* signal attenuation, rephrase itself? directly related to the amount of agent which remains in the tissue. These data yield information about tissue perfusion and blood volume at the ROI; malignant tumors primarily show a rapid wash-in and wash-out? of contrast in enhancement time curve.

Many groups are working to generate parametric response maps (PRM) for post tumor-response evaluation; these models use peak enhancement or wash-in rate models based on pixelby-pixel calculation. The resulting datasets and response maps have been used to develop sophisticated statistical models for the physiological response of tumors to therapy [14].

\section{Diffusion weighted imaging (DWI)}

Diffusion-weighted imaging (DWI) has been proven to be one of the most useful tools in tumor response evaluation, and cohort studies show its potential in radiation treatment planning.

DWI pulse sequences can generate contrast based on the diffusion of water molecules in biological tissues. In healthy tissue, the diffusion of water molecules is restricted, governed by their interaction with many obstacles, including macromolecules, fibers, and membranes. The DWI signal can be used to map out the tissue architecture of normal and tumor tissue. One of the principle factors affecting tissue dynamics is cell density, as high cell density causes more water-restricted diffusion: generally, when a tumor has high cell density, the water diffusion is more restricted.

DWI signal is sensitive to water mobility and cellularity in different tissues. The DWI signal benefits from the use of two equally large opposing symmetric diffusion gradient pulses to generate the contrast on moving water molecules. The first gradient pulse induces a phase shift in water molecules within the tissue; after rephasing by the second diffusion pulse, the amount of signal remaining depends on phase difference between mobile and stationary water molecules. Hence, in low cell density tissues water 
molecules generate more DWI signal; however, the reverse effect has been seen in high density cells and when the tumor is highly glandular or has significant necrosis; under those conditions, lower signal intensity or complete signal loss is observed [15-17].

The strength and duration of diffusion gradients can be adjusted (b-values) to generate another quantitative parameter from DWI images: the apparent diffusion coefficient (ADC). The $\mathrm{ADC}$, in units of $\mathrm{mm} 2 \cdot \mathrm{s}-1$, has been used to assess water diffusion through tissue. The ADC map can be derived directly by acquiring at least two images with different $b$ values (usually $b=0$ and $b=1000$ $\mathrm{s} \cdot \mathrm{mm}^{2}$ ). For example, mean ADC values for benign and malignant head and neck lesions are $1.51 \cdot 10^{-3} \mathrm{~mm}^{2} \cdot \mathrm{s}-1$ and $1.07 \cdot 10^{-3} \mathrm{~mm}^{2} \cdot \mathrm{s}-1$, respectively.

The value of ADC has been evident in the clinic, and it is in increasing use due to its ability to monitor treatment apoptosis, cell swelling, tumor lysis and necrosis [2]. The standard and functional MRI pulse sequences, including DWI and ADC maps, can be used for tumor detection, staging and defining the microscopic tissue structure of tumor tissue. Using DWI for tumor detection and contouring in treatment planning system is made more difficult by its geometrical distortion. DWI pulse sequences have been developed to address this issue, and even beyond that, to enable DWI for imaging gated and online MRI-linac applications. Traditional DWI images have deformable registration relative to other MRI sequence images or CT images, and must be compared to them to define the extra potential volume of recurrence in gross target volume (GTV) in head and neck cancer cases [18] or riskadaptive boosting [19].

\section{Magnetic Resonance Spectroscopic Imaging (MRSI)}

In vivo single or multi-voxel MRSI can provide information about tissue metabolites. The commonly used nuclei are Hydrogen $\left({ }^{1} \mathrm{H}\right)$, Phosphorus-31 $\left({ }^{31} \mathrm{P}\right)$, Fluorine-19 $\left({ }^{19} \mathrm{~F}\right)$, and Carbon-13 $\left({ }^{13} \mathrm{C}\right)$. After defining the ROI or voxel location on a tumor or in normal tissue, the MRI spectrum can be generated, which contains peaks at specific frequencies (chemical shifts), providing the MRI fingerprint of different metabolites. Each chemical possesses unique electron shielding based on its structure, which will affect the local magnetic field and resonance frequency, or chemical shift, thus allowing identification [2].

MRSI can be used in a variety of ways: as single voxel spectroscopy (SVS) with spatial resolution of 1-8 cm, which is very time consuming, but can be used quantitatively; as multi-voxel chemical shift imaging (CSI) is able? to measure larger volumes of tissue, and capable of division into smaller voxels during post processing; and as 2D MRSI-CSI, a technique with a longer acquisition time and post-processing.

Many studies show that the ratio of N-Acetylaspartate (NAA) to choline (related to cell turnover) serves a marker of brain tumors that can be evaluated using MRSI. In the prostate, the ratio of citrate to choline, or choline to creatine has been used with good results. Interestingly, lactate has been found in drug-resistant tumors, and has been called the hypoxic metabolite. Lactate can currently be found in prostate tumors using high field MRI. It also has been found in lipids in head and neck and brain tumors [20]. Interpretation of MRSI data requires a high level of expertise and their acquisition requires use of a very homogenous B0 field with higher order shimming at the ROI. The use of these data for treatment planning is still challenging. Additionally, hypoxic zones may be chronic or acute, and may change position over time, making imaging them more challenging $[21,22]$.

\section{Discussion}

MRI has been shown to be superior to CT in target volume definition for several diagnoses, and is now widely in routine clinical use. Its superior soft tissue contrast compared to CT and the possibilities for functional imaging using DWI and DCE-MRI imaging are contributing factors to our belief that MRI will be the primary imaging modality for delineation in radiotherapy in the future. The development of integrated MRI and treatment units can push this development even further, and make MRI the sole imaging modality for large groups of patients, that is, both for definition of treatment targets and organs at risk, and for positioning of the patient at treatment. Here we will discuss a few treatment sites which have been shown to benefit from this technology.

\section{Brain}

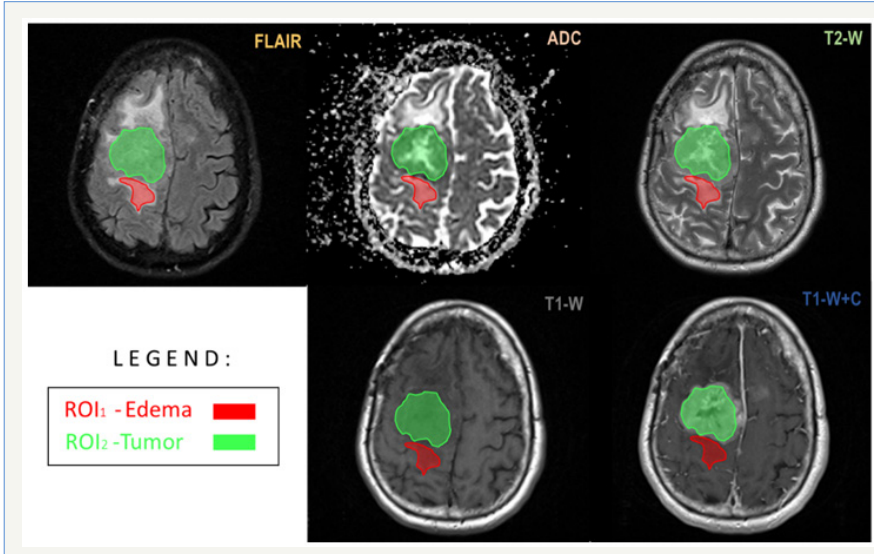

Figure 1: Volumetric brain multiparametric MR imaging used for parametric response map construction after chemoradiation in a 55-year-old male patient with a right temporal GBM. The axial FLAIR image demonstrates segmentation (red and green) of the region of FLAIR hyper-intensity corresponding to the area of edema/ tumor infiltration. Notice the segmented enhancement that has been confirmed and segmented on the ADC map, T1W, T1WI post-contrast, and T2W.

The brain was one of the first sites to be imaged by MRI for RTP. MRI provides better tumor and normal tissue delineation than CT; therefore, it is more frequently used in treatment planning systems for tumor contouring in high dose focal brain radiotherapy such as fractionated radiotherapy (RT), Fractionated stereotactic radiotherapy (FSRT), and stereotactic radiosurgery (SRS) (Figure 1 \& 2). We scan patients with MRI using a standard 16 or 32 channel $\mathrm{RF}$ head coil with a thermoplastic immobilizer or stereotactic radiosurgery frame in treatment position, and perform the register of the MRI image set to the CT simulation image set in the treatment 
planning process. MRI can be used for guided radiotherapy during diagnostic and post-treatment response measurements.

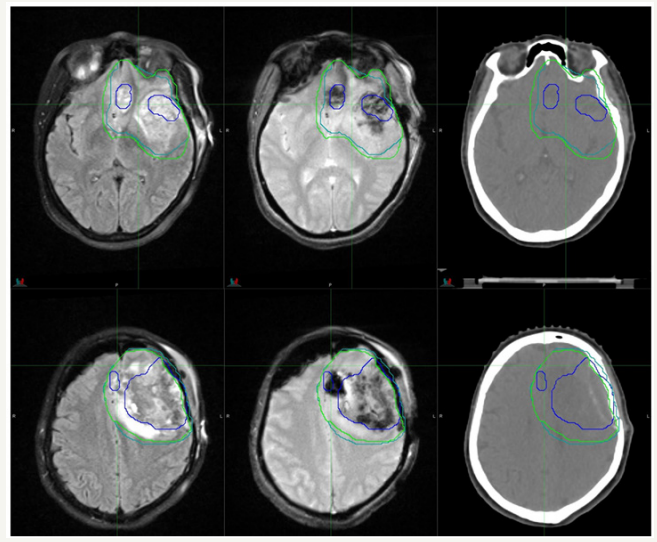

Figure 2: VMAT Plan for a GBM patient Left: diagnostic T1 sequence. Center: treatment T2 Flair sequence; Right: Contours on CT for planning using MRI images: Dark Blue $=$ Pre-Op GTV; Green = T2 Flair target $($ Post-Op $)$; Aqua = GTV-T2 Flair (Pre-Op).

Clinically, high-grade brain tumor boundaries such as those in glioblastoma (GBM) can be highlighted on contrast-enhanced T1-weighted images such as those acquired with T1-weighted magnetization-prepared radio-frequency pulses and rapid gradient-echo (MPRAGE) as well as regular T2-weighted images [23]. Fast fluid-attenuated inversion recovery (FLAIR) images have also been found useful to define tumor edema and possible infiltration [24]. For high grade brain tumors such as GBM T1weighed high resolution isotropic 3D images acquired postcontrast are being used for tumor contouring and determination of gross target volume (GTV), while a series of T2-weighed and FLAIR images define the clinical target volume (CTV). For brain metastasis and low grade or benign brain tumors there is no tumor invasion to normal brain tissue; therefore, post-contrast high resolution 3D T1-weighted images are sufficient for tumor delineation, especially for SRS treatments (Figure 3).
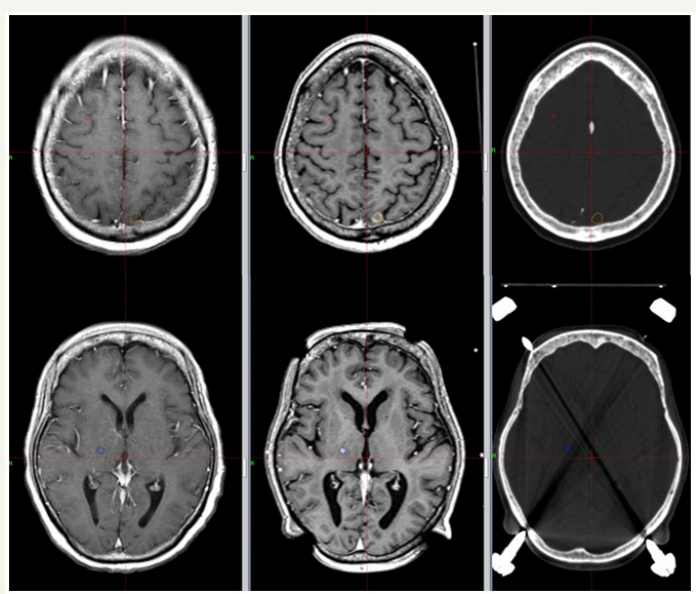

Figure 3: Brain metastasis. Left: diagnostic T1 spin echo (C + FC) sequence; Center: treatment T1-MPRAGE sequence; Right:. Contours delineate two separate lesions on CT images for planning using MRI images.
Magnetic Resonance Angiography (MRA) is used to define nidus in treatment of arteriovenous malformations (AVMs) [25,26]. Three-dimensional steady state gradient echo sequences such as constructive interference in steady state (CISS) have applications to define the involved trigeminal nerve in SRS trigeminal treatment. CISS signal is heavily T2 weighted, and cerebral spinal fluid around the brain stem shows hypointen sesignal, while normal brain tissue and cranial nerves show no signal. This feature allows contouring of the involved trigeminal nerve root as it emanates from the brainstem [27]. Recently he have shown that using modified high b-value DWI images to define the hypercellular component, and corresponding adjustment to prescribed 95\%-isodose volume (PDV) will improves rates of progression-free survival (PFS). Functional MRI (fMRI) is being used regularly for stereotactic brain surgery, and of late many research groups are investigating applications of fMRI during RTP to define and contour OARs [1127].

Optimal targeting of GBM for RT using CT and MRI remains challenging because of the tumor's infiltrative nature. Traditionally, two RT target volumes are created on the planning CT as follows: one to define enhancing tissue and the resection cavity on contrastenhanced T1-weighted MRI, and another to define signal changes on a T2-weighted and FLAIR sequence. However, histological analyses indicate that these sequences fail to capture the true extent of disease because of the presence of infiltrative, non-enhancing tumor tissue [28-32]. In addition, anatomic MRI provides limited information on the physiological state and biochemical activity of a viable tumor [12]. The inability to accurately identify active tumor tissue using conventional neuroimaging remains a limiting factor in GBM RT planning.

MRSI, as mentioned above, is a molecular imaging technique that maps the metabolism of native small molecules in tumor regions in vivo without exogenous tracers [33,34]. Studies have shown that the addition of MRS to tumor targeting allows identification of unique metabolic regions of interest with significant clinical consequence (i.e., regions of tumor progression) [35-39]. Moreover, recently, it was shown that the choline to $\mathrm{N}$-Acetylaspartate ratio (Cho/NAA), a marker describing glial cell proliferation, correlates with tumor infiltration, progression- free survival, and contrast enhancement at recurrence in GBM [40]. Thus, Cho/NAA regions of interest deserve consideration during treatment planning, and may be of use if effectively integrated into image guidance.

Technical limitations have hindered the use of MRSI in RT planning. Sequence-specific limitations, including poor spatial resolution and limited brain coverage, obscure the margins of metabolic abnormalities and prevent visualization of infiltration into the surrounding brain tissue. This hinders accurate and complete delineation of tumor infiltration, curbing the value of metabolic data in treatment planning for GBM. In addition, there is a paucity of clinical decision support software for the analysis, visualization, and management of spectroscopic data. Consequently, spectroscopic processing requires a skilled user to evaluate spectra and manually transfer data containing maps with a nominal voxel 
size of $4.4 \times 4.4 \times 5.6 \mathrm{~mm}^{3}$ at acquisition; post-processing yields an effective voxel volume of $1 \mathrm{~mL}$ [41-43].

\section{Prostate}

MRI is well established in contouring the prostate due to its excellent soft tissue resolution (Figure 4), ability to determine the apex of the prostate with high accuracy, and avoidance of CT artifacts in patients with hip implants $[43,44]$.

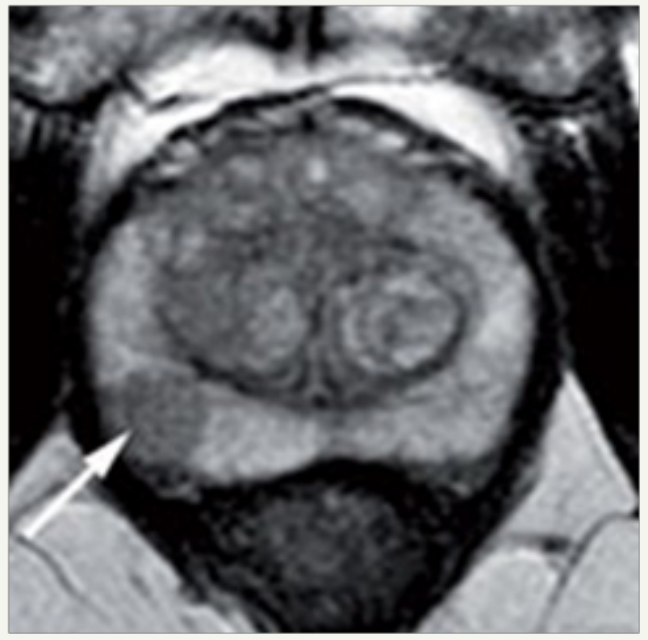

Figure 4: T2 MRI showing prostate cancer as $1 \mathrm{~cm}$ hypointensity in the right peripheral zone.

\section{Lung}

MRI lung imaging is challenging, but optimized MRI sequences can provide sufficient contrast and temporal resolution to accurately map a tumor and its location. This information provides a tremendous advantage for 3D planning and motion management [45]. Stereotactic body radiotherapy (SBRT) is rapidly becoming an alternative to surgery for the treatment of early-stage, non-small cell lung cancer patients. Lung SBRT is administered in a hypofractionated, conformal manner, delivering targeted high doses to the treatment area. To avoid normal-tissue toxicity, it is crucial to limit the exposure of nearby healthy OAR.

Current image-guided radiotherapy strategies for lung SBRT are mostly based on X-ray imaging modalities. Although still in its infancy, magnetic resonance imaging (MRI) guidance for lung SBRT is not exposure-limited and MRI promises to improve crucial softtissue contrast. Looking beyond anatomical imaging, functional MRI is expected to assist in treatment decisions and adaptations in the future. A range of acquisition techniques has been employed to determine tumor size and the extent of tumor growth with MRI, including standard T1- or T2-weighted acquisitions, 2D cine MRI, and single-shot Fast Spin Echo (FSE) MRI. T2-weighted, dynamic contrast-enhanced (DCE), and DWI MRI are promising in their ability to distinguish between benign and malignant tumors [46].

Identifying disease spread to lymph nodes is difficult using PET-CT due to its limited spatial resolution. Several MRI sequences have been investigated to stage mediastinal and hilar lymph node metastases [47]. A recent meta-analysis of twelve studies has concluded that MRI offers improvements in sensitivity and accuracy over PET-CT [48]. It remains to be seen whether MRI can complement the ubiquitous FDG-PET for diagnosis and staging of lesions. The higher spatial resolution of MRI could prove beneficial for staging and detection of smaller nodes and metastases that might go undetected in PET-CT.

Like 4D CT and 4D CBCT - albeit not as established - 4D MRI can be used to evaluate periodic anatomical changes due to breathing. 4D MRI can be either obtained based on repeated acquisition of slices covering the whole volume and respiratory cycle [49-51] or using a continuous 3D acquisition with sophisticated reconstruction techniques [52-54]. While 4D MRI based on 2D acquisitions is more readily available, the $3 \mathrm{D}$ techniques offer higher resolution and allow for full 3D distortion correction.

A conceptual limitation of 4D scans is that they average over several respiratory cycles, and thus cannot accurately depict baseline shifts of breathing motion [55]. MRI can be utilized to acquire 2D cine MR images that provide a better visualization of the patient's breathing, especially when it comes to capturing irregularities. Combining 4D MRI with 2D cine MRI using a principle component analysis (PCA)-based approach allows generation of 3D deformable vector fields with a temporal resolution of $476 \mathrm{~ms}$ [51]. In the future, this temporally resolved observation of tumor motion could be used to define adequate treatment margins.

\section{Head and Neck}

The common MRI pulse sequences and their application in head and neck cancer include DCE-MRI and DWI with apparent diffusion coefficient (ADC) maps to define tumor boundary and tissue properties; the BOLD technique (Blood Oxygen Level Dependent) for hypoxic areas; and ultra-fast cine MRI sequences to tag and track tumor and normal tissue for 3D planning. Transient functional MRI studies and scans will allow personalized radiation treatment, and are a strategy proposed for hypo-fractionation and online guided radiotherapy [56-60].

Functional MRI is an important tool for understanding the dynamics of tumor response to radiotherapy, and has potential applications in adapting and individualizing treatment. To adapt biological models to data from functional imaging, treatment response and resistant volumes must be surveyed., a goal achievable with ADC maps from DWI. Once a predictive model of tumor response based on the functional image is built, a new optimization method will be possible. The integration of functional MRI in RTP and treatment response evaluation has attracted the interest of research groups worldwide [61] including the assembly of hybrid MRI-linac machines [62]

With specialized RF coils, MRI can be performed in treatment positions, improving sensitivity and specificity (Figure 5). MRI is especially useful in patients with dental implants where CT images are poor due to artefacts, and is more useful than CT for contouring base-of-skull tumors with possible intracranial involvement. 


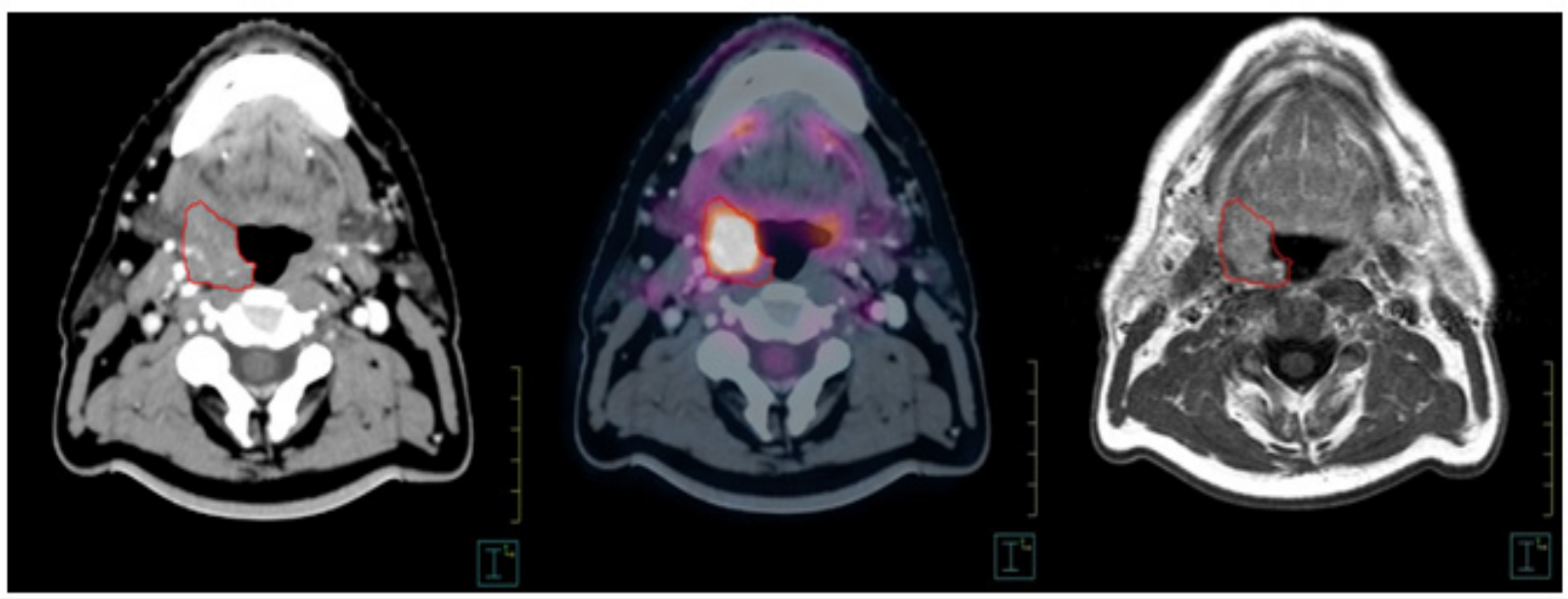

Figure 5: Base-of-tongue tumor imaged with different techniques. Left: CT simulator; Center: PET-FDG; Right: T1-weighted postcontrast MRI.

\section{Breast}

DCE-MRI is a routine screening tool for breast cancer (Figure 6). Some research groups report using ultra small particle iron oxides (USPIO) to detect involved lymph nodes as small as $2 \mathrm{~mm}$ on T2*-weighted MRI pulse sequences. This information can be used during treatment planning to spare uninvolved lymph nodes, has the potential to support conservative breast surgery and more targeted radiation therapy, thereby reducing the side effects of radiation and surgery $[63,64]$.

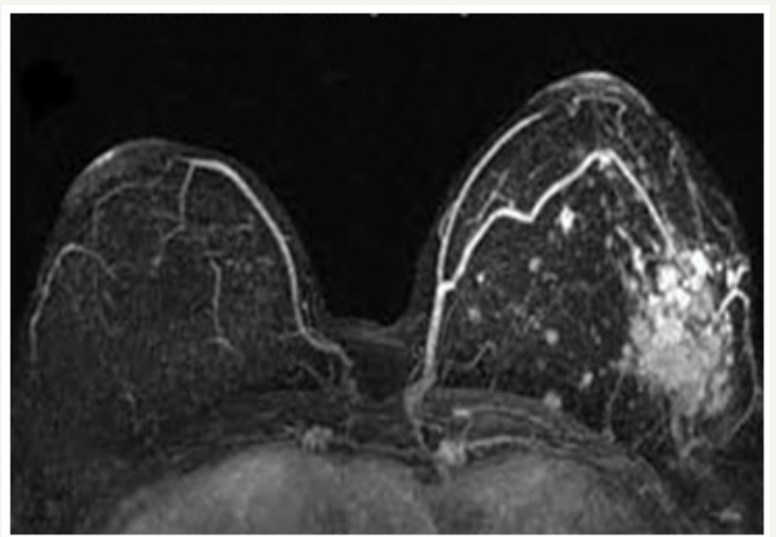

Figure 6: Breast cancer screening with MRI. Contrast enhanced subtraction MRI reveals a mass in left breast.

\section{Cervix}

MRI is a well-established modality for cervical cancer diagnostics and routine checkups. More specifically, MRI scanning, both with and without contrast agent, is used to look for parametrial and lymph node involvement, using high resolution T1- and T2weighted images. MRI-guided high dose radiation rate (HDR) brachytherapy is in increasing use, enabled by MRI compatible and distortion-free MRI pulse sequences [65].

\section{Conclusion}

Current advances in MRI machine design and online and offline 3D and 2D geometrical distortion correction algorithms have opened new directions in the use of MRI for RTP. MRI images can not only be directly used in the treatment planning process to delineate tumor and normal tissues, but also to provide useful functional information about post-tumor response? tumor response post radiation? Functional MRI can be used in adaptive radiotherapy, and MRI study of tumor biomarkers can help identify late effects of radiation treatment. Analysis of these biomarkers will help to individualize radiation treatments and develop understanding of radiobiology. We believe continued use of MRI in radiotherapy will create new opportunities for customized MRI machines, instruments, and MRI pulse sequences for guided radiotherapy and radiation treatment planning, leading to improved health outcomes.

\section{Acknowledgement}

I would like to thank all of my colleagues in radiation oncology and radiology for their support.

\section{References}

1. Tubiana M (2009) Can we reduce the incidence of second primary malignancies occurring after radiotherapy? A critical review. Radiother Oncol 91: 4-15.

2. Desar I, Van Herpen C, Van Laarhoven H, Barentsz J, Oyen W, et al. (2009) Beyond RECIST: molecular and functional imaging techniques for evaluation of response to targeted therapy. Cancer Treat. Rev. 35(4): 309-321.

3. Fallone B, Carlone M, Murray B, Rathee S, Stanescu T, et al. (2007) TU-CM100F-01: Development of a linac-MRI system for real-time. ART Med Phys 34(6): 2547.

4. Raaymakers B, Lagendijk J, Overweg J, Kok J, Raaijmakers A, et al. (2009) Integrating a 1.5 T MRI scanner with a 6 MV accelerator: proof of concept. Phys Med Biol 54: N229.

5. Oborn B, Metcalfe P, Butson M, Rosenfeld A, Keall P (2012) Electron contamination modeling and skin dose in $6 \mathrm{MV}$ longitudinal field MRIgRT: Impact of the MRI and MRI fringe field. Med Phys 39(2): 874890.

6. Raaymakers B, Raaijmakers A, Lagendijk J (2008) Feasibility of MRI guided proton therapy: magnetic field dose effects. Phys Med Biol 53(20): 5615-5622.

7. Mazaheri Y, Shukla Dave A, Muellner A, Hricak H (2011) MRI of the prostate: Clinical relevance and emerging applications. J Magn Reson Imaging 33: 258-274. 
8. Philpotts LE (2011) Comprehensive breast imaging 2010. Seminars in Roentgenology 46(1): 7-17.

9. Hounsfield GN (1973) Computerized transverse axial scanning (tomography): Part 1. Description of system. Br J Radiol 46(552): 10161022 .

10.P Metcalfe, T Kron, P Hoban (2007) PP of radiotherapy X from linear accelerators Metcalfe, The physics of radiotherapy x-rays and electrons. Medical Physics Pub.

11. Garcia Alvarez R, Liney GP, Beavis AW (2006) Repeatability of functional MRI for conformal avoidance radiotherapy planning. J Magn Reson Imaging 23(2): 108-114.

12. Kovacs A, Hadjiev J, Lakosi F, Antal G, Vandulek C, et al. (2009) Dynamic MR based analysis of tumor movement in upper and mid lobe localized lung cancer. Pathol Oncol Res 15: 269-277.

13. Ogawa S, Lee TM, Nayak AS, Glynn P (1990) Oxygenation-sensitive contrast in magnetic resonance image of rodent brain at high magnetic fields. Magn Reson Med 14(1): 68-78.

14.Tofts PS, Brix G, Buckley DL, Evelhoch JL, Henderson E, et al. (1999) Estimating kinetic parameters from dynamic contrast-enhanced T1weighted MRI of a diffusable tracer: standardized quantities and symbols. J Magn Reson Imaging 10(3): 223-232.

15.Vandecaveye V, De Keyzer F, Nuyts S, Deraedt K, Dirix P, et al. (2007) Detection of head and neck squamous cell carcinoma with diffusion weighted MRI after (chemo) radiotherapy: correlation between radiologic and histopathologic findings. Int J Radiat Oncol Biol Phys 67(4): 960-971.

16.Srinivasan A, Dvorak R, Perni K, Rohrer S, Mukherji S (2008) Differentiation of benign and malignant pathology in the head and neck using 3T apparent diffusion coefficient values: early experience. AJNR Am J Neuroradiol 29(1): 40-44

17. Bourne R, Kurniawan N, Cowin G, Sved P, Watson G (2011) 16 T Diffusion microimaging of fixed prostate tissue: preliminary findings. Magn Reson Med 66(1): 244-247

18. Ling CC, Humm J, Larson S, Amols H, Fuks Z, et al. (2000) Towards multidimensional radiotherapy (MD-CRT): biological imaging and biological conformality. Int J Radiat Oncol Biol Phys 47: 551-560.

19. Kim Y, Tomé WA (2006) Risk-adaptive optimization: selective boosting of high-risk tumor sub volumes. Int J Radiat Oncol Biol Phys 66(5): 15281542 .

20.Star Lack J, Spielman D, Adalsteinsson E, Kurhanewicz J, Terris DJ, et al (1998) In vivo lactate editing with simultaneous detection of choline creatine, NAA, and lipid singlets at $1.5 \mathrm{~T}$ using PRESS excitation with applications to the study of brain and head and neck tumors. J Magn Reson 133: 243-254.

21. Kwock L, Smith JK, Castillo M, Ewend MG, Collichio F, et al. (2006) Clinica role of proton magnetic resonance spectroscopy in oncology: brain breast, and prostate cancer. Lancet Oncol 7(10): 859-868.

22.Frahm J, Haase A, Matthaei D (1986) Rapid NMR imaging of dynamic processes using the FLASII technique. Magn Reson Med 3: 321-327.

23. Nelson SJ, Cha S (2003) Imaging glioblastoma multiforme. Cancer J 9: 134.

24.Essig M, Schlemmer HP, Tronnier V, Hawighorst H, Wirtz R, et al. (2011) Fluid-attenuated inversion-recovery MR imaging of gliomatosiscerebri Eur Radiol 11(2): 303-308.

25.Tomé WA, Mehta MP, Meeks SL, Buatti JM (2002) Fractionated stereotactic radiotherapy: present and future. Technol Cancer Res Treat 1: 153-172.

26. Petereit D, Mehta M, Turski P, Levin A, Strother C, et al. (1993) Treatment of arteriovenous malformations with stereotactic radiosurgery employing both magnetic resonance angiography and standard angiography as a database. Int J Radiat Oncol Biol Phys 25(2): 309-313.
27. Richards GM, Bradley KA, Tomé WA, Bentzen SM, Resnick DK, et al. (2005) Linear accelerator radiosurgery for trigeminal neuralgia. Neurosurgery 57(6): 1193-2000.

28. Hamstra DA, Galbán CJ, Meyer CR, Johnson TD, Sundgren PC, et al. (2008) Functional diffusion map as an early imaging biomarker for high-grade glioma: correlation with conventional radiologic response and overall survival. J Clin Oncol 26(2): 3387-3394.

29. Mardor Y, Pfeffer R, Spiegelmann R, Roth Y, Maier SE, et al. (2003) Early detection of response to radiation therapy in patients with brain malignancies using conventional and high b-value diffusion-weighted magnetic resonance imaging. J Clin Oncol 21(6): 1094-1100.

30. Kovács Á, Tóth L, Glavák C, Liposits G, Hadjiev J, et al. (2011) Integrating functional MRI information into conventional 3D radiotherapy planning of CNS tumors. Is it worth it? J Neurooncol 105(3): 629-637.

31. Burger PC, Dubois PJ, Schold SC, Smith KR, Odom GL, et al. (1983) Computerized tomographic and pathologic studies of the untreated, quiescent, and recurrent glioblastoma multiforme. J Neurosurg 58(2): 159-169.

32. McKnight TR, von demBussche MH, Vigneron DB, Lu Y, Berger MS, et al. (2002) Histopathological validation of a three-dimensional magnetic resonance spectroscopy index as a predictor of tumor presence. J Neurosurg 97(4): 794-802.

33. Narayana A, Chang J, Thakur S, Huang W, Karimi S, et al. (2007) Use of MR spectroscopy and functional imaging in the treatment planning of gliomas. Br J Radiol 80(953): 347-354.

34. Farace P, Giri MG, Meliado G, Amelio D, Widesott L, et al. (2011) Clinical target volume delineation in glioblastomas: pre-operative versus postoperative/pre-radiotherapy MRI. Br J Radiol 84(999): 271-278.

35. Burger PC, Heinz ER, Shibata T, Kleihues P (1988) Topographic anatomy and CT correlations in the untreated glioblastoma multiforme. J Neurosurg 68(5): 698-704.

36. Cao Y, Sundgren PC, Tsien CI, Chenevert TT, Junck L (2006) Physiologic and metabolic magnetic resonance imaging in gliomas. J Clin Oncol 24(8): 1228-1235.

37. Nelson SJ (2003) Multivoxel magnetic resonance spectroscopy of brain tumors. Mol. Cancer Ther 2(5): 497-507.

38. Nelson SJ, Graves E, Pirzkall A, Li X, Antiniw Chan A, et al. (2002) In vivo molecular imaging for planning radiation therapy of gliomas: an application of 1H MRSI. J Magn Reson Imaging 16(4): 464-476.

39. Einstein DB, Wessels B, Bangert B, Fu P, Nelson AD, et al. (2012) Phase II trial of radiosurgery to magnetic resonance spectroscopy-defined high-risk tumor volumes in patients with glioblastoma multiforme. Int J Radiat Oncol Biol Phys 84(3): 668-674.

40. Pirzkall A, Li X, Oh J, Chang S, Berger MS, et al. (2004) 3D MRSI for resected high-grade gliomas before RT: tumor extent according to metabolic activity in relation to MRI. Int J Radiat Oncol Biol Phys 59(1) 126-137.

41. Ken S, Vieillevigne L, Franceries X, Simon L, Supper C, et al. (2013) Integration method of 3D MR spectroscopy into treatment planning system for glioblastoma IMRT dose painting with integrated simultaneous boost. Radiat Oncol 8: 1.

42. Parra NA, Maudsley AA, Gupta RK, Ishkanian F, Huang K, et al. (2014) Volumetric spectroscopic imaging of glioblastoma multiforme radiation treatment volumes. Int J Radiat Oncol Biol Phys 90(2): 376-84.

43. Rosewall T, Kong V, Vesprini D, Catton C, Chung P, et al. (2009) Prostate delineation using CT and MRI for radiotherapy patients with bilateral hip prostheses. Radiother Oncol 90(3): 325-330.

44. Milosevic M, Voruganti S, Blend R, Alasti H, Warde P, et al. (1998) Magnetic resonance imaging (MRI) for localization of the prostatic apex: comparison to computed tomography (CT) and urethrography. Radiother Oncol 47(3): 277-284. 
45. Blackall J, Ahmad S, Miquel M, McClelland J, Landau D, et al. (2006) MRIbased measurements of respiratory motion variability and assessment of imaging strategies for radiotherapy planning. Phys Med Biol 51: 4147 4169.

46. Puderbach M, Hintze C, Ley S, Eichinger M, Kauczor HU, et al. (2007) MR imaging of the chest: a practical approach at 1.5T. Eur J Radiol 64 (3): 345-355.

47. Hasegawa I, Eguchi K, Kohda E, Tanami Y, Mori T, Hatabu H, et al. (2003) Pulmonary hilar lymph nodes in lung cancer: assessment with 3D-dynamic contrast-enhanced MR imaging. Eur JRadiol 45(2): 129-134

48. Peerlings J, Troost EGC, Nelemans PJ, Cobben DCP, de Boer JCJ, et al. (2016) The diagnostic value of MR imaging in determining the lymph node status of patients with nonsmall cell lung cancer: a meta-analysis. Radiology 281(1): 86-98.

49. Tryggestad E, Flammang A, Han-Oh S, Hales R, Herman J, et al. (2013) Respiration-based sorting of dynamic MRI to derive representative 4D-MRI for radiotherapy planning. Med Phys 40(5): 051909.

50. Liu Y, Yin FF, Czito BG, Bashir MR, Cai J (2015) T2-weighted fourdimensional magnetic resonance imaging with result-driven phase sorting. Med Phys 42(8): 4460-4471.

51. Stemkens B, Tijssen RHN, de Senneville BD, Lagendijk JJW, van den Berg CAT (2016) Image-driven, model-based 3D abdominal motion estimation for MR-guided radiotherapy. Phys Med Biol 61(14): 5335-5355.

52. Feng L, Grimm R, Block KT, Chandarana H, Kim S, et al. (2014) Goldenangle radial sparse parallel MRI: combination of compressed sensing, parallel imaging, and golden-angle radial sampling for fast and flexible dynamic volumetric MRI. MagnReson Med. 72(3): 707-717.

53. Rank CM, Heuer T, Buzan MTA, Wetscherek A, Freitag MT, et al. (2017) $4 \mathrm{D}$ respiratory motion-compensated image reconstruction of freebreathing radial MR data with very high undersampling. Magn Reson Med 77(3): 1170-1183.

54. Mickevicius NJ, Paulson E (2017) Investigation of under sampling and reconstruction algorithm dependence on respiratory correlated 4D-MRI for online MR- guided radiation therapy. Phys Med Biol 62(8): 2910 2921

55. Tryggestad E, Flammang A, Hales R, Herman J, Lee J, et al. (2013) 4D tumor centroid tracking using orthogonal 2D dynamic MRI: implications for radiotherapy planning. Med Phys 40(9): 091712.
56. Khoo VS, Joon DL (2006) New developments in MRI for target volume delineation in radiotherapy. Br J Radiol 79: S2-15.

57. Kim S, Loevner L, Quon H (2009) Diffusion-weighted magneticresonance imaging for predicting and detecting early response to chemoradiation therapy of squamous cell carcinomas of the head and neck. Clin. Cancer Rev 15(3): 986-994.

58. Vandecaveye V, De Keyzer F, Nuyts S (2007) Detection of head and neck squamous cell carcinoma with diffusion weighted MRI after (chemo) radiotherapy: correlation between radiologic and histopathologic findings. Int J Radiat Oncol Biol Phys 67: 960-971.

59.Koh DM, Collins DJ (2007) Diffusion weighted MRI in the body: applications and challenges in oncology. Am J Roentgenol 188: 16221635.

60. Yankeelov TE, Lepage $M$, Chakravarthy A (2007) Integration of quantitative DCE-MRI and ADC mapping to monitor treatment response in human breast cancer: initial results. Magn Reson Imaging 25(1): 1-13.

61.López Medina A, Aramburu D, Mera M (2013) ARTFIBio Project: quantifying tumour response voxel by voxel. Second ESTRO Forum Book of Abstracts. Elsevier, Geneva, Switzerland.

62. Raaymakers BW, Lagendijk JJ, Overweg J (2009) Integrating a 1.5 T MRI scanner with a 6 MV accelerator: proof of concept. Phys Med Biol 54(12): 229-237.

63. Kirby AM, Yarnold JR, Evans PM, Morgan VA, Schmidt MA, et al. (2009) Tumor bed delineation for partial breast and breast boost radiotherapy planned in the prone position: what does MRI add to X-ray CT localization of titanium clips placed in the excision cavity wall? Int J Rad Oncol Biol Phys 74: 1276-1282.

64. Mack MG, Balzer JO, Straub R, Eichler K, Vogl TJ (2002) Super paramagnetic iron oxide-enhanced MR imaging of head and neck lymph nodes1. Radiology 222: 239-244.

65. Haie-Meder C, Pötter R, Van Limbergen E, Briot E, De Brabandere, et al. (2005) Recommendations from gynaecological (GYN) GEC-ESTRO working group: concepts and terms in 3D image based 3D treatment planning in cervix cancer brachytherapy with emphasis on MRI assessment of GTV and CTV. Radiother Oncol 74(3): 235-245. 